\title{
Composite Goldstone dark matter: experimental predictions from the lattice
}

\author{
Ari Hietanen, ${ }^{a}$ Randy Lewis, ${ }^{b}$ Claudio Pica ${ }^{a}$ and Francesco Sannino ${ }^{a}$ \\ ${ }^{a} C P^{3}$ and the Danish IAS, \\ University of Southern Denmark, Campusvej 55, DK-5230 Odense M, Denmark \\ ${ }^{b}$ Department of Physics and Astronomy, York University, \\ Toronto, M3J 1P3, Canada \\ E-mail: hietanen@cp3-origins.net, randy.lewis@yorku.ca, \\ pica@cp3-origins.net, sannino@cp3-origins.net
}

AbSTRACT: We study, via lattice simulations, the nonperturbative dynamics of $\mathrm{SU}(2)$ gauge theory with two fundamental Dirac flavors. The model can be used simultaneously as a template for composite Goldstone boson dark matter and for breaking the electroweak symmetry dynamically. We compute the form factor, allowing us to estimate the associated electromagnetic charge radius. Interestingly we observe that the form factor obeys vector meson dominance even for the two color theory. We finally compare the model predictions with dark matter direct detection experiments. We find that the composite Goldstone boson dark matter cross section is constrained by the most stringent direct-detection experiments. Our results are a foundation for quantitative new composite dynamics relevant for model building, and are of interest to current experiments.

Keywords: Lattice QCD, Beyond Standard Model, Technicolor and Composite Models ArXiv EPRINT: 1308.4130 


\section{Contents}

1 Introduction 1

2 The lattice method 3

3 Relationships among form factors 5

$\begin{array}{lll}4 & \text { The lattice results } & 7\end{array}$

5 Photon-dark matter form factor: the basics 11

6 Adding the composite Higgs $\quad 13$

$\begin{array}{llr}7 & \text { Conclusions } & 16\end{array}$

$\begin{array}{ll}\text { A Lattice measurements } & 18\end{array}$

\section{Introduction}

Unveiling the nature of dark matter (DM) constitutes a fundamental problem in physics. DM plays an important role in large scale structure formation as well as the evolution of the Universe. Several earth and space based experiments are searching for DM to study its properties.

Starting from the simple observation that the bulk of the ordinary matter is composite, i.e. is made by neutrons and protons, it is justified and intriguing to explore the paradigm according to which also DM has a composite nature.

Composite Higgs models, such as the contemporary Technicolor models, present relevant examples in which the model can simultaneously address the naturalness problems of the SM and offer well-motivated composite DM states. Composite DM states in these models can be heavy, typically of the order a few $\mathrm{TeV}$ [1-3], when identified with the composite fermions of the theory, or light, i.e. with masses ranging from a few $\mathrm{GeV}$ to hundreds of $\mathrm{GeV}$ if identified with the (pseudo) Goldstone [4]. Several asymmetric DM candidates appeared in the literature [1-11]. An interesting variation on the main composite Higgs theme is the one according to which the composite Higgs is also a (pseudo) Goldstone boson by Kaplan and Georgi [12]. A unified description of composite Higgs models is given in [13].

However, so far, composite Goldstone DM phenomenology relied solely on the symmetries of the underlying gauge theory and effective Lagrangians descriptions. While these approaches are useful, an estimate of the form factors dictating the interactions, and associated physics, between the DM candidate and ordinary matter is essential to guide the experimental searches. Furthermore, due to the composite nature of the DM states, the 
knowledge of the energy dependence of the form factors allows to study and relate the DM properties in different energy regimes ranging from a few $\mathrm{keV}$ to hundreds of $\mathrm{GeV}$.

Here we consider a template of composite Goldstone boson DM [8] investigated on the lattice in $[14,15]$, namely an $\mathrm{SU}(2)$ gauge theory with two fundamental fermion flavors. We view this theory as the kernel from which more elaborate models can grow. For example, there are extensions that show how a $125 \mathrm{GeV}$ scalar can emerge [16]. The USQCD collaboration highlighted this lattice theory in a recent white paper [17] and studied the effects of additional fermions in [18]. Other groups have reported results at strong coupling [19], results with nonzero chemical potential [20-24], and results with chiral lattice fermions [25]. Dark matter candidates from nuclei in this lattice theory were discussed in [26, 27]. Dark matter in the related $\mathrm{SU}(3)$ and $\mathrm{SU}(4)$ lattice theories were considered in [28] and [29] respectively. Our minimal template has the appeal to address simultaneously electroweak symmetry breaking and the origin of a naturally-light DM candidate [8].

The template is an $\mathrm{SU}(2)$ gauge theory with two fundamental fermion flavors, named $u$ and $d$. This action has a global SU(4) symmetry, and the lattice simulations of ref. [14] showed that it is dynamically broken to $\mathrm{Sp}(4)$, thereby producing five Goldstone bosons. Three of these are eaten by the $W^{ \pm}$and $Z$ bosons; the remaining pair of Goldstones is the DM candidate and its antiparticle. Depending on the cross section for annihilation into standard model fields one can have a symmetric (i.e. thermal relic density), asymmetric, or a mixed scenario [11]. An exact Goldstone boson would-be massless but, like the pions of QCD, the DM candidate can acquire a small mass from explicit symmetry breaking through new interactions breaking the original $\mathrm{SU}(4)$ symmetry to $\mathrm{SU}_{L}(2) \times \mathrm{SU}_{R}(2) \times \mathrm{U}(1)$ while keeping the $u$ and $d$ massless. The effective Lagrangian operator was constructed in [8] and corresponds to an effective four-fermion interaction. However, as recently pointed out in [13], standard model radiative corrections alone are sufficient to give mass to the would-be Goldstone Boson. The present model allows us to study the interaction between composite DM and ordinary matter by determining the associated electric dipole moment.

The light DM limit was originally introduced to explore models of interfering DM $[5,30,31]$ useful to alleviate the tension between the experimental observations by DAMA/LIBRA [32] and the limits set by XENON100 [33, 34] and CDMS [35]. However with the very constraining results by LUX [36] it has become increasingly harder to reconcile these anomalies. We will therefore assume here a very conservative attitude and compare our results only with the most severe exclusion results from LUX, XENON100 and SuperCDMS [37].

The present paper is organized as follows. Section 2 explains how lattice computations of the form factors can be performed. Section 3 derives relationships among the form factors of the five Goldstone bosons. Section 4 presents the numerical results of our lattice simulations and provides evidence that vector meson saturation for the form factors applies even in the case of the two color theory. Section 5 combines the lattice results to determine the electroweak form factor and the associated DM proton cross section. The effect of Higgs exchange and the direct comparison with the experimental data is presented in section 6 . Section 7 contains our conclusions. 


\section{The lattice method}

In the continuum, the Lagrangian for our technicolor template is

$$
\mathcal{L}=-\frac{1}{4} F_{\mu \nu}^{a} F^{a \mu \nu}+\bar{u}\left(i \gamma^{\mu} D_{\mu}-m_{u}\right) u+\bar{d}\left(i \gamma^{\mu} D_{\mu}-m_{d}\right) d
$$

which can be discretized in the familiar way to arrive at a Wilson action,

$$
\begin{aligned}
S_{W}= & \frac{\beta}{2} \sum_{x, \mu, \nu}\left(1-\frac{1}{2} \operatorname{Re} \operatorname{Tr} U_{\mu}(x) U_{\nu}(x+\hat{\mu}) U_{\mu}^{\dagger}(x+\hat{\nu}) U_{\nu}^{\dagger}(x)\right)+\sum_{x} \bar{\psi}(x)\left(4+m_{0}\right) \psi(x) \\
& -\frac{1}{2} \sum_{x, \mu}\left(\bar{\psi}(x)\left(1-\gamma_{\mu}\right) U_{\mu}(x) \psi(x+\hat{\mu})+\bar{\psi}(x+\hat{\mu})\left(1+\gamma_{\mu}\right) U_{\mu}^{\dagger}(x) \psi(x)\right)
\end{aligned}
$$

where $U_{\mu}$ is the gauge field and $\beta$ the gauge coupling in conventional lattice notation. $\psi$ is the doublet of $u$ and $d$ fermions, and $m_{0}$ is the $2 \times 2$ diagonal mass matrix.

Mesons will couple to local operators of the form

$$
\begin{aligned}
\mathcal{O}_{\bar{u} d}^{(\Gamma)}(x) & =\bar{u}(x) \Gamma d(x) \\
\mathcal{O}_{\bar{d} u}^{(\Gamma)}(x) & =\bar{d}(x) \Gamma u(x) \\
\mathcal{O}_{\bar{u} u \pm \bar{d} d}^{(\Gamma)}(x) & =\frac{1}{\sqrt{2}}(\bar{u}(x) \Gamma u(x) \pm \bar{d}(x) \Gamma d(x)),
\end{aligned}
$$

where $\Gamma$ denotes any product of Dirac matrices. Baryons (which are diquarks in this two-color theory) will couple to local operators of the form

$$
\begin{aligned}
\mathcal{O}_{u d}^{(\Gamma)}(x) & =u^{T}(x)\left(-i \sigma^{2}\right) C \Gamma d(x), \\
\mathcal{O}_{d u}^{(\Gamma)}(x) & =d^{T}(x)\left(-i \sigma^{2}\right) C \Gamma u(x), \\
\mathcal{O}_{u u \pm d d}^{(\Gamma)}(x) & =\frac{1}{\sqrt{2}}\left(u^{T}(x)\left(-i \sigma^{2}\right) C \Gamma u(x) \pm d^{T}(x)\left(-i \sigma^{2}\right) C \Gamma d(x)\right),
\end{aligned}
$$

where the Pauli structure $-i \sigma^{2}$ acts on color indices while the charge conjugation operator $C$ acts on Dirac indices.

A photon can couple to a local vector operator such as $\mathcal{O}_{\bar{u} u \pm \bar{d} d}^{\left(\gamma_{\mu}\right)}$ which becomes a conserved current in the continuum limit but is not conserved in the lattice theory. In studies of the electroweak form factors, it is advantageous to work directly with the lattice conserved currents,

$$
\begin{aligned}
V_{\mu}^{u}(x) & =\frac{1}{2} \bar{u}(x+\hat{\mu})\left(1+\gamma_{\mu}\right) U_{\mu}^{\dagger}(x) u(x)-\frac{1}{2} \bar{u}(x)\left(1-\gamma_{\mu}\right) U_{\mu}(x) u(x+\hat{\mu}), \\
V_{\mu}^{d}(x) & =\frac{1}{2} \bar{d}(x+\hat{\mu})\left(1+\gamma_{\mu}\right) U_{\mu}^{\dagger}(x) d(x)-\frac{1}{2} \bar{d}(x)\left(1-\gamma_{\mu}\right) U_{\mu}(x) d(x+\hat{\mu}),
\end{aligned}
$$

that are easily combined to produce the electromagnetic current,

$$
V_{\mu}(x)=\frac{1}{2} V_{\mu}^{u}(x)-\frac{1}{2} V_{\mu}^{d}(x) .
$$




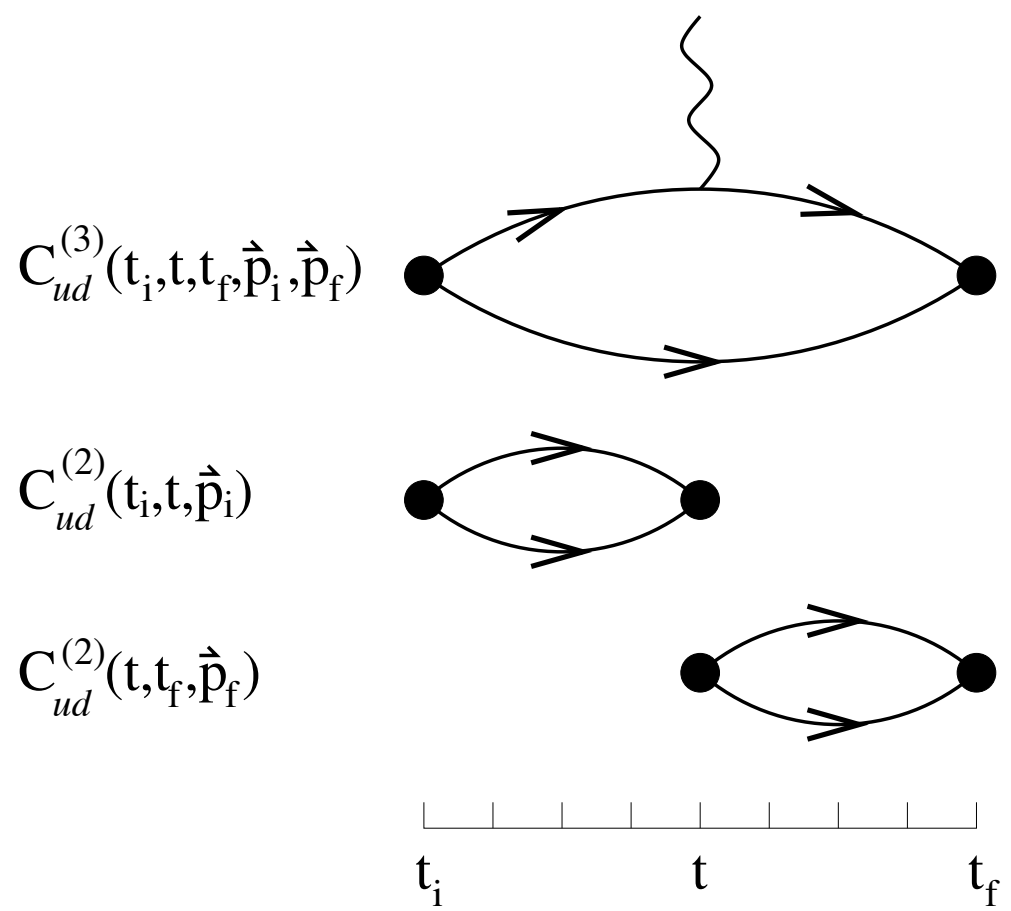

Figure 1. The three correlation functions analyzed in a simultaneous fit to determine the mass and form factor of a Goldstone boson. The central time $t$ is varied throughout the range $t_{i}<t<t_{f}$. The outgoing Goldstone boson momentum is chosen to be $\vec{p}_{f}=\overrightarrow{0}$ in our simulations.

A three-point correlation function that probes the elastic form factor of the DM candidate is

$$
C_{u d}^{(3)}\left(t_{i}, t, t_{f}, \vec{p}_{i}, \vec{p}_{f}\right)=\sum_{\vec{x}_{i}, \vec{x}, \vec{x}_{f}} e^{-i\left(\vec{x}_{f}-\vec{x}\right) \cdot \vec{p}_{f}} e^{-i\left(\vec{x}-\vec{x}_{i}\right) \cdot \vec{p}_{i}}\left\langle 0\left|\mathcal{O}_{u d}^{\left(\gamma_{5}\right)}\left(x_{f}\right) V_{\mu}(x) \mathcal{O}_{u d}^{\left(\gamma_{5}\right) \dagger}\left(x_{i}\right)\right| 0\right\rangle
$$

where $\vec{x}$ denotes the spatial 3 -vector within the 4 -vector $x$. A two-point correlation function represents particle propagation,

$$
C_{u d}^{(2)}\left(t_{i}, t_{f}, \vec{p}\right)=\sum_{\vec{x}_{i}, \vec{x}_{f}} e^{-i\left(\vec{x}_{f}-\vec{x}_{i}\right) \cdot \vec{p}}\left\langle 0\left|\mathcal{O}_{u d}^{\left(\gamma_{5}\right)}\left(x_{f}\right) \mathcal{O}_{u d}^{\left(\gamma_{5}\right) \dagger}\left(x_{i}\right)\right| 0\right\rangle
$$

Two methods have been used for the lattice analysis, as has been done from the earliest dynamical study of the pion form factor in SU(3) QCD [38]. One method is to perform a simultaneous fit to the three correlation functions shown pictorially in figure 1 . In particular, these correlation functions must be fit to their expected hadronic forms:

$$
\begin{aligned}
C_{u d}^{(3)}\left(t_{i}, t, t_{f}, \vec{p}_{i}, \vec{p}_{f}\right) & =\sum_{n_{i}} \sum_{n_{f}} Z_{n_{f}} \frac{e^{-\left(t_{f}-t\right) E_{n_{f}}\left(\vec{p}_{f}\right)}}{2 E_{n_{f}}\left(\vec{p}_{f}\right)}\left\langle n_{f}\left(\vec{p}_{f}\right)\left|V_{\mu}(0)\right| n_{i}\left(\vec{p}_{i}\right)\right\rangle \frac{e^{-\left(t-t_{i}\right) E_{n_{i}}\left(\vec{p}_{i}\right)}}{2 E_{n_{i}}\left(\vec{p}_{i}\right)} Z_{n_{i}}^{*}, \\
C_{u d}^{(2)}\left(t_{i}, t_{f}, \vec{p}\right) & =\sum_{n}\left|Z_{n}\right|^{2} \frac{e^{-\left(t_{f}-t_{i}\right) E_{n}(\vec{p})}}{2 E_{n}(\vec{p})} .
\end{aligned}
$$


In principle the sums include all hadrons having the quantum numbers of the operator $\mathcal{O}_{u d}^{\left(\gamma_{5}\right)}$, but in practice only the lightest few hadrons will be resolved by typical lattice data if $t_{i}$ and $t_{f}$ are sufficiently far apart on the lattice. In our simulations $C^{(3)}$ is dominated by the ground state, i.e. the Goldstone boson of interest (generically named $\Pi$ ), but excited states are still observed in the pair of $C^{(2)}$ correlators. Therefore we can fit to

$$
\begin{aligned}
C_{u d}^{(3)}\left(t_{i}, t, t_{f}, \vec{p}_{i}, \vec{p}_{f}\right) & =\left|Z_{\Pi}\right|^{2} \frac{e^{-\left(t_{f}-t\right) E_{\Pi}\left(\vec{p}_{f}\right)}}{2 E_{\Pi}\left(\vec{p}_{f}\right)} \frac{e^{-\left(t-t_{i}\right) E_{\Pi}\left(\vec{p}_{i}\right)}}{2 E_{\Pi}\left(\vec{p}_{i}\right)} F_{\Pi}\left(Q^{2}\right)\left(p_{i}+p_{f}\right)_{\mu}, \\
C_{u d}^{(2)}\left(t_{i}, t_{f}, \vec{p}\right) & =\left|Z_{\Pi}\right|^{2} \frac{e^{-\left(t_{f}-t_{i}\right) E_{\Pi}(\vec{p})}}{2 E_{\Pi}(\vec{p})}+\sum_{\text {excited } n}\left|Z_{n}\right|^{2} \frac{e^{-\left(t_{f}-t_{i}\right) E_{n}(\vec{p})}}{2 E_{n}(\vec{p})}
\end{aligned}
$$

where we have used the standard definition of the form factor $F_{\Pi}\left(Q^{2}\right)$,

$$
\begin{aligned}
\left\langle\Pi\left(\vec{p}_{f}\right)\left|V_{\mu}(0)\right| \Pi\left(\vec{p}_{i}\right)\right\rangle & =F_{\Pi}\left(Q^{2}\right)\left(p_{i}+p_{f}\right)_{\mu}, \\
Q^{2} & =\left(\vec{p}_{f}-\vec{p}_{i}\right)^{2}-\left(E_{\Pi}\left(\vec{p}_{f}\right)-E_{\Pi}\left(\vec{p}_{i}\right)\right)^{2} .
\end{aligned}
$$

For any chosen lattice momentum, the fit parameters are the energies $E_{\Pi}$ and $E_{n}$, the coefficients $\left|Z_{\Pi}\right|^{2}$ and $\left|Z_{n}\right|^{2}$, and the form factor $F_{\Pi}\left(Q^{2}\right)$.

Notice that our fitting functions are not periodic in the Euclidean time direction. Because a form factor calculation has three widely-spaced times, $t_{i}, t$, and $t_{f}$, it is more economical to use a Dirichlet boundary condition in the time direction for fermions. Therefore the fitting functions described above are the correct ones for our simulations.

The second method used for the lattice analysis, which gives results that are in complete agreement with the first method, is known as the ratio method. This second method uses an explicit formula for the form factor, valid for $t_{i} \ll t \ll t_{f}$ :

$$
F_{\Pi}\left(Q^{2}\right)=\frac{C_{U D}^{(3)}\left(t_{i}, t, t_{f}, \vec{p}_{i}, \vec{p}_{f}\right) C_{U D}^{(2)}\left(t_{i}, t, \vec{p}_{f}\right)}{C_{U D}^{(2)}\left(t_{i}, t, \vec{p}_{i}\right) C_{U D}^{(2)}\left(t_{i}, t_{f}, \vec{p}_{f}\right)}\left(\frac{2 E_{\Pi}\left(\vec{p}_{f}\right)}{E_{\Pi}\left(\vec{p}_{i}\right)+E_{\Pi}\left(\vec{p}_{f}\right)}\right) .
$$

It is straightforward to derive this expression from the preceding equations. The ratio method is very convenient because all $Z_{n}$ have canceled away, and the ratio $E_{\Pi}\left(\vec{p}_{i}\right) / E_{\Pi}\left(\vec{p}_{f}\right)$ is easy to obtain from the lattice two-point functions. All that remains is to fit the ratio to a constant for each value of $Q^{2}$. Another pleasant feature of eq. (2.20) is that the only two-point function that extends all the way from $t_{i}$ to $t_{f}$ has momentum $\vec{p}_{f}$. Because we always choose $\vec{p}_{f}=\overrightarrow{0}$, our simulations will provide a precise numerical value for this factor in the ratio.

\section{Relationships among form factors}

To determine what signal our DM candidate would induce to direct detection experiments, we estimate the electromagnetic form factors. The $u$ and $d$ fermions in our action have electroweak charges that are constrained by anomaly cancellation: they form a left-handed weak doublet, right-handed weak singlets, and have electric charges $Q_{u}=+1 / 2$ and $Q_{d}=$ $-1 / 2$. Neither fermion carries QCD color. The five Goldstone bosons have valence structure 
$\bar{u} d, \bar{d} u, \frac{1}{\sqrt{2}}(\bar{u} u-\bar{d} d), u d$ and $\bar{u} \bar{d}$. Because it is symmetric under $u \leftrightarrow d$, the DM candidate $u d$ has no electroweak elastic form factors if there is no isospin breaking. Only the two electrically-charged Goldstones will have form factors in that case. If a source of isospin breaking appears the electroweak elastic form factors will not vanish for the DM candidate $u d$, and they will be related to the form factors of the charged Goldstones. Such a source of isospin breaking is naturally expected to occur in Nature given that is already present for the ordinary quarks, and moreover they are welcome because they can be used to further diminish, or eliminate, the tension with the precision data. To mimic this source of isospin breaking on the lattice we will simply assume two different explicit masses for the up and down fermions.

Our lattice study can therefore follow the methods used for early quenched studies of $\mathrm{SU}(2)$ gauge theory [47-49] and recent dynamical studies of $\mathrm{SU}(3)$ gauge theory [38, 40-46], with the difference that our $\mathrm{SU}(2)$ fermions are dynamical.

The five Goldstone bosons form a multiplet within the remaining $\mathrm{Sp}(4)$ global symmetry, but that symmetry is not respected by electroweak interactions. There are also deviations arising from $m_{u} \neq m_{d}$. Here we derive some of the connections between correlation functions of the Goldstone bosons.

To begin, we adapt a derivation provided in ref. [14]

$$
\begin{aligned}
C_{u d}^{(2)}\left(t_{i}, t_{f}, \vec{p}\right) & =\sum_{\vec{x}_{i}, \vec{x}_{f}} e^{-i\left(\vec{x}_{f}-\vec{x}_{i}\right) \cdot \vec{p}}\left\langle 0\left|\mathcal{O}_{u d}^{\left(\gamma_{5}\right)}\left(x_{f}\right) \mathcal{O}_{u d}^{\left(\gamma_{5}\right) \dagger}\left(x_{i}\right)\right| 0\right\rangle \\
& =\sum_{\vec{x}_{i}, \vec{x}_{f}} e^{-i\left(\vec{x}_{f}-\vec{x}_{i}\right) \cdot \vec{p}} \operatorname{Tr}\left(u^{T}\left(x_{f}\right)\left(-i \sigma^{2}\right) C \gamma_{5} d\left(x_{f}\right) \bar{d}\left(x_{i}\right) \gamma_{0} \gamma_{5}^{\dagger} C^{\dagger}\left(-i \sigma^{2}\right)^{\dagger} \gamma_{0}^{T} \bar{u}^{T}\left(x_{i}\right)\right) \\
& =\sum_{\vec{x}_{i}, \vec{x}_{f}} e^{-i\left(\vec{x}_{f}-\vec{x}_{i}\right) \cdot \vec{p}} \operatorname{Tr}\left(\bar{u}\left(x_{f}\right) \gamma_{5} d\left(x_{f}\right) \bar{d}\left(x_{i}\right) \gamma_{0} \gamma_{5}^{\dagger} \gamma_{0} u\left(x_{i}\right)\right) \\
& =C_{\bar{u} d}^{(2)}\left(t_{i}, t_{f}, \vec{p}\right)
\end{aligned}
$$

where we have made use of two properties of the charge conjugation operator:

$$
\begin{aligned}
\gamma^{\mu T} & =-C \gamma^{\mu} C^{\dagger} \\
{[u(y) \bar{u}(x)]^{T} } & =C\left(-i \sigma^{2}\right) u(x) \bar{u}(y) C^{\dagger}\left(-i \sigma^{2}\right)^{\dagger} .
\end{aligned}
$$

Similar derivations lead to the following relations among three-point correlation functions,

$$
\begin{aligned}
C_{u d}^{(3)}\left(t_{i}, t, t_{f}, \vec{p}_{i}, \vec{p}_{f}\right) & =T^{u}-T^{d}, \\
C_{\overline{u d}}^{(3)}\left(t_{i}, t, t_{f}, \vec{p}_{i}, \vec{p}_{f}\right) & =-T^{u}+T^{d}, \\
C_{u \bar{d}}^{(3)}\left(t_{i}, t, t_{f}, \vec{p}_{i}, \vec{p}_{f}\right) & =T^{u}+T^{d}, \\
C_{\bar{u} d}^{(3)}\left(t_{i}, t, t_{f}, \vec{p}_{i}, \vec{p}_{f}\right) & =-T^{u}-T^{d}, \\
C_{\bar{u} u+\bar{d} d}^{(3)}\left(t_{i}, t, t_{f}, \vec{p}_{i}, \vec{p}_{f}\right) & =0,
\end{aligned}
$$

where

$$
T^{X}=\sum_{\vec{x}_{i}, \vec{x}, \vec{x}_{f}} e^{-i\left(\vec{x}_{f}-\vec{x}\right) \cdot \vec{p}_{f}} e^{-i\left(\vec{x}-\vec{x}_{i}\right) \cdot \vec{p}_{i}}\left\langle 0\left|\mathcal{O}_{u d}^{\left(\gamma_{5}\right)}\left(x_{f}\right) V_{\mu}^{X}(x) \mathcal{O}_{u d}^{\left(\gamma_{5}\right) \dagger}\left(x_{i}\right)\right| 0\right\rangle
$$


For the special case of $m_{u}=m_{d}$, we find $T^{u}=T^{d}$ so only the charged Goldstones, $\bar{u} d$ and $\bar{d} u$, have a nonzero form factor. In the general case of $m_{u} \neq m_{d}$, we see that the DM candidate $u d$ (and its antiparticle) also has a form factor.

Lattice simulations could in principle determine $T^{u}$ and $T^{d}$ in the general case, but they contain contributions from quark-disconnected diagrams that would require significant computational resources. Lattice simulations with $m_{u}=m_{d}$ are more manageable, but then $T^{u}=T^{d}$ so there is no DM form factor in that case.

There is an explicit relationship between $T^{u}$ and $T^{d}$ in the large $N_{c}$ limit. In that limit hadronic resonances become narrow, so $T^{u}$ and $T^{d}$ are each written as a sum over vector meson poles [50-52]. In practice those sums are dominated by the lightest vector mesons. Perhaps surprisingly, this large $N_{c}$ result has long been known to work rather well for QCD despite the seemingly small value of $N_{c}=3$. For example, the $\pi^{+}$form factor is dominated by $\rho^{0}$ meson exchange and the $K^{+}$form factor is dominated by $\rho^{0}$ and $\phi$ meson exchange,

$$
\begin{aligned}
F_{\pi^{+}}\left(Q^{2}\right) & \approx \frac{2}{3}\left(\frac{m_{\rho}^{2}}{m_{\rho}^{2}+Q^{2}}\right)+\frac{1}{3}\left(\frac{m_{\rho}^{2}}{m_{\rho}^{2}+Q^{2}}\right), \\
F_{K^{+}}\left(Q^{2}\right) & \approx \frac{2}{3}\left(\frac{m_{\rho}^{2}}{m_{\rho}^{2}+Q^{2}}\right)+\frac{1}{3}\left(\frac{m_{\phi}^{2}}{m_{\phi}^{2}+Q^{2}}\right) .
\end{aligned}
$$

QCD also contains an example that exactly parallels our $m_{u} \neq m_{d}$ effects: the neutral kaon has a nonzero form factor arising from $m_{d} \neq m_{s}$. The experimental determination of the neutral kaon charge radius [53] is dominated by the difference between $\rho^{0}$ and $\phi$ meson exchanges,

$$
\begin{aligned}
F_{K^{0}}\left(Q^{2}\right) & \approx-\frac{1}{3}\left(\frac{m_{\rho}^{2}}{m_{\rho}^{2}+Q^{2}}\right)+\frac{1}{3}\left(\frac{m_{\phi}^{2}}{m_{\phi}^{2}+Q^{2}}\right), \\
\left\langle r^{2}\right\rangle_{K^{0}} & =-\left.6 \frac{d F_{K^{0}}}{d Q^{2}}\right|_{Q^{2}=0}
\end{aligned}
$$

If the large $N_{c}$ result were also applicable to our $N_{c}=2$ technicolor template, then lattice determinations of the vector meson masses would provide estimates of all Goldstone form factors. Moreover, the dark matter form factors would be related to $W^{ \pm}$form factors. In the following section we will perform a lattice simulation of the Goldstone form factor in the $m_{u}=m_{d}$ limit, i.e. $T^{u}+T^{d}$, and show that the large $N_{c}$ result does indeed hold to a good accuracy in our $N_{c}=2$ theory.

\section{The lattice results}

The numerical work in this paper is based on the same configurations generated in [15]. A complete analysis of 500 configurations at $\left(\beta, m_{0}\right)=(2.2,-0.72)$ provides a first result for the form factor. To consider discretization effects an analysis of 300 configurations at $\left(\beta, m_{0}\right)=(2.0,-0.947)$ is performed. To study chiral extrapolation effects, an analysis of 300 configurations at $\left(\beta, m_{0}\right)=(2.2,-0.75)$ is performed. All ensembles were created 


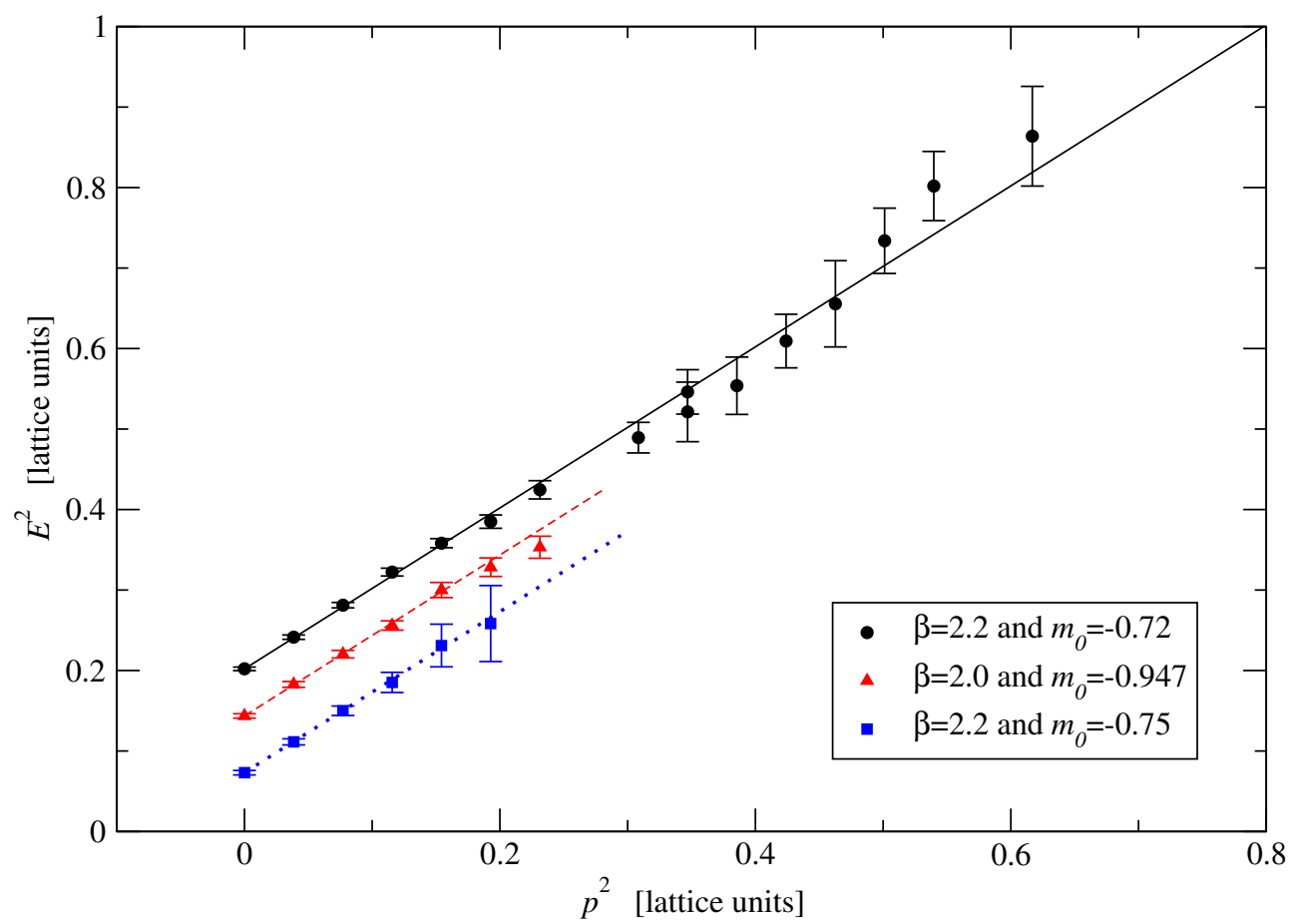

Figure 2. Squared energies of Goldstone bosons as functions of $p^{2}=p_{x}^{2}+p_{y}^{2}+p_{z}^{2}$. Straight lines are $m_{\Pi}^{2}+p^{2}$ for the measured lattice mass $m_{\Pi}$.

with the HiRep code [54] for fully-dynamical plaquette-action SU(2) gauge theory with two flavors of mass-degenerate Wilson fermions.

Extraction of the form factor requires the energies of Goldstone bosons that are moving across the lattice. There is a direct relationship in the continuum,

$$
E^{2}=m^{2}+p^{2}
$$

and also on the lattice

$$
\begin{aligned}
\hat{E}^{2} & =4 \sinh ^{2}(m / 2)+\hat{p}^{2}, \\
\hat{E} & \equiv 2 \sinh (E / 2), \\
\hat{p}^{2} & \equiv 4 \sum_{i=1}^{3} \sin ^{2}\left(p_{i} / 2\right) .
\end{aligned}
$$

Figure 2 shows three straight lines that represent the continuum relation; the only input for those lines is the Goldstone mass because their slopes are completely determined by kinematics. Direct lattice computations of the energy of a moving Goldstone boson are also shown. Note that lattice discretization provides access to

$$
\vec{p}=\frac{2 \pi}{L}\left(k_{x} \hat{x}+k_{y} \hat{y}+k_{z} \hat{z}\right)
$$

where $L=32$ and we use $0 \leq k_{i} \leq 3$. Since the data presented in figure 2 lie on the continuum lines, there is no indication of any discretization errors. This conclusion is true 


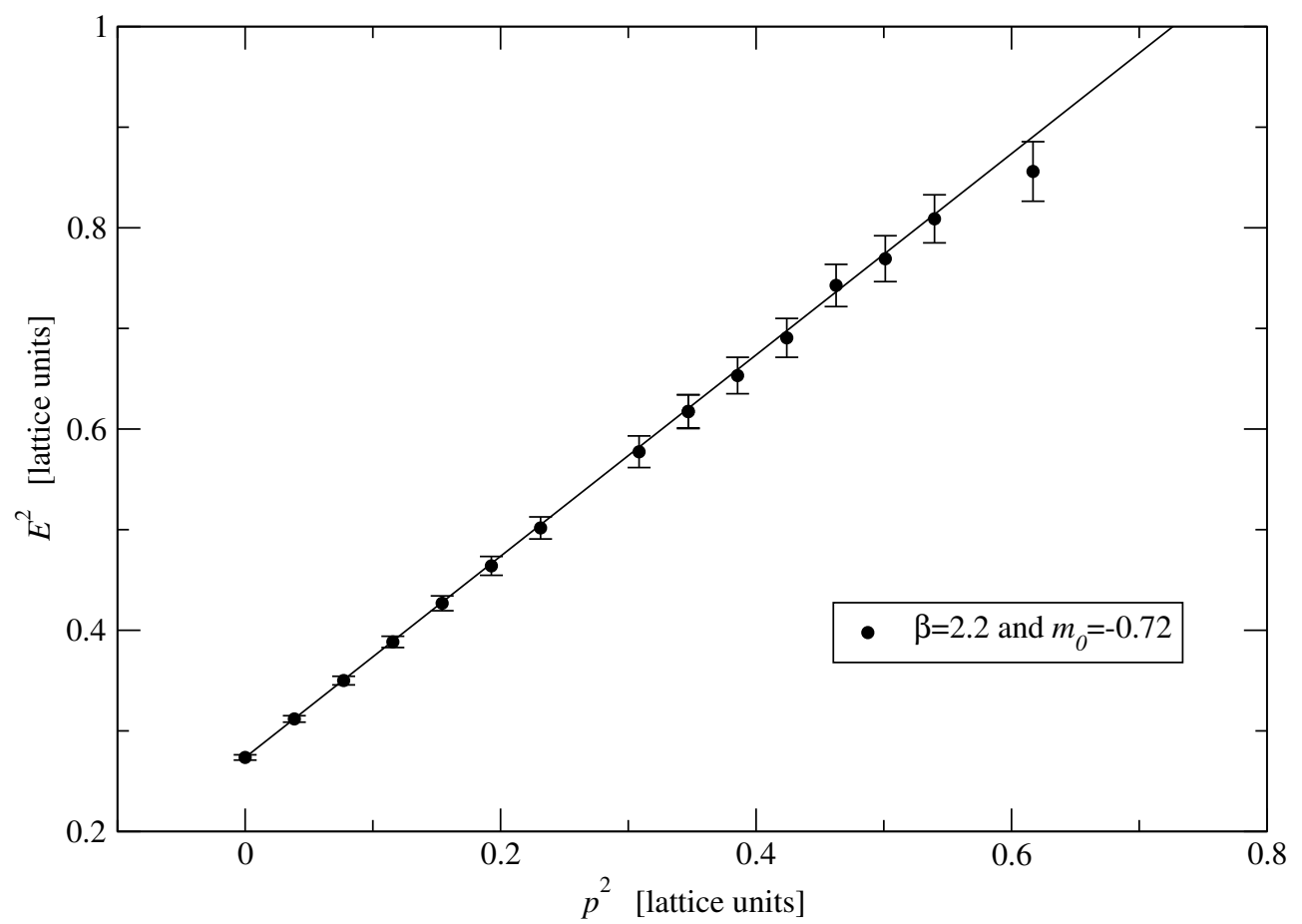

Figure 3. Squared energy of the vector meson as a function of $p^{2}=p_{x}^{2}+p_{y}^{2}+p_{z}^{2}$, for $\left(\beta, m_{0}\right)=$ $(2.2,-0.72)$. The straight line is $m_{V}^{2}+p^{2}$ for the measured lattice mass $m_{V}$.

of all three data sets up to $p^{2} \sim 0.6$, though only one data set was shown for the full range to avoid cluttering the plot.

More precise lattice data are obtained for the vector meson, in part because of the ability to average over all three polarizations. Figure 3 shows the agreement with continuum expectations for one data set. The mass agrees with ref. [14] and the momentum dependence agrees with the continuum line. The other two data sets are displayed in figure 4 with the coarser lattice extended as far as $p^{2} \approx 0.6$. For comparison, that same data set is compared to the lattice expectation in figure 5 where the required agreement is seen for all momentum values. Though discretization effects are modest, we will ensure self-consistency by using lattice relations rather than continuum relations when analyzing the form factor.

The vector meson is of interest to the present work because the Goldstone boson form factor is expected to exhibit vector meson dominance. The straight lines in figure 4 indicate that two of our ensembles have nearly-equal vector meson masses in lattice units, suggesting that their form factors should also be similar, although figure 2 shows that their Goldstone masses are not equal.

We choose the outgoing Goldstone to be at rest in our form factor computations, so momentum flows from the incoming Goldstone to the photon coupling. All momentum directions are averaged for each configuration; for example, form factors with $\left(k_{x}, k_{y}, k_{z}\right)=(1,0,2),(1,2,0),(2,0,1),(2,1,0),(0,1,2)$ and $(0,2,1)$ in eq. $(4.5)$ are all computed and averaged to help reduce statistical errors. We use Dirichlet boundary conditions in the time direction for fermions in the measurements, meaning that fermions do not propa- 


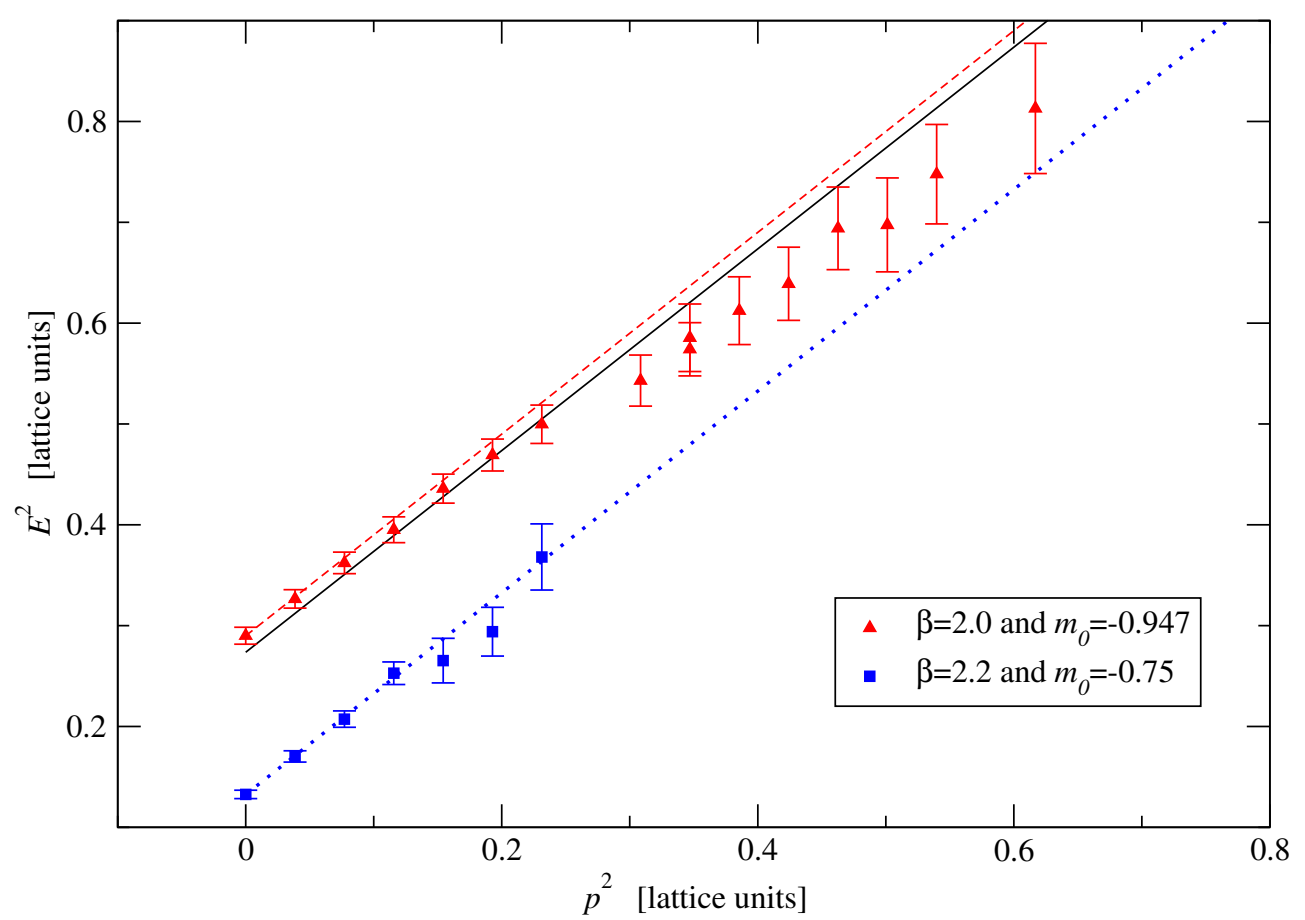

Figure 4. Squared energies of the vector meson as functions of $p^{2}=p_{x}^{2}+p_{y}^{2}+p_{z}^{2}$, for $\left(\beta, m_{0}\right)=$ $(2.0,-0.947)$ and $\left(\beta, m_{0}\right)=(2.2,-0.75)$. Straight lines are $m_{V}^{2}+p^{2}$ for the measured lattice mass $m_{V}$ : dashed line for $\left(\beta, m_{0}\right)=(2.0,-0.947)$, dotted line for $\left(\beta, m_{0}\right)=(2.2,-0.75)$, and (for comparison) solid line for $\left(\beta, m_{0}\right)=(2.2,-0.72)$.

gate beyond the lattice's temporal boundaries. However, the configurations were generated using periodic boundary conditions in time directions. The Goldstone creation operator is placed at the fifth time step from the lattice's left edge $\left(t_{i}=4\right)$ and the annihilation operator is placed at the fifth from the right $\left(t_{f}=27\right)$.

As an example of lattice data for the form factor, figure 6 shows the raw form factor data for the right-hand side of eq. (2.20) with one particular momentum in the $\left(\beta, m_{0}\right)=$ $(2.2,-0.72)$ ensemble. There is a broad range of Euclidean times between $t_{i}$ and $t_{f}$ where the ratio is indeed constant, allowing the form factor to be read from the plot. When a similar plot is made for vanishing momentum, the form factor is exactly equal to unity due to our use of the conserved vector current which obeys the corresponding lattice WardTakahashi identity.

The four-momentum transfer is defined by

$$
q=\left(\vec{p}_{f}-\vec{p}_{i}, E_{f}-E_{i}\right)
$$

and putting that into the continuum dispersion relation, eq. (4.1), gives

$$
Q^{2} \equiv-q^{2}=\left(\vec{p}_{f}-\vec{p}_{i}\right)^{2}-\left(E_{f}-E_{i}\right)^{2}
$$

while putting it into the lattice dispersion relation, eq. (4.2), gives

$$
\hat{Q}^{2} \equiv-q^{2}=-4 \operatorname{arcsinh}^{2} \sqrt{\sinh ^{2}\left(\frac{E_{f}-E_{i}}{2}\right)-\sum_{j=x, y, z} \sin ^{2}\left(\frac{\left(p_{f}-p_{i}\right)_{j}}{2}\right)}
$$




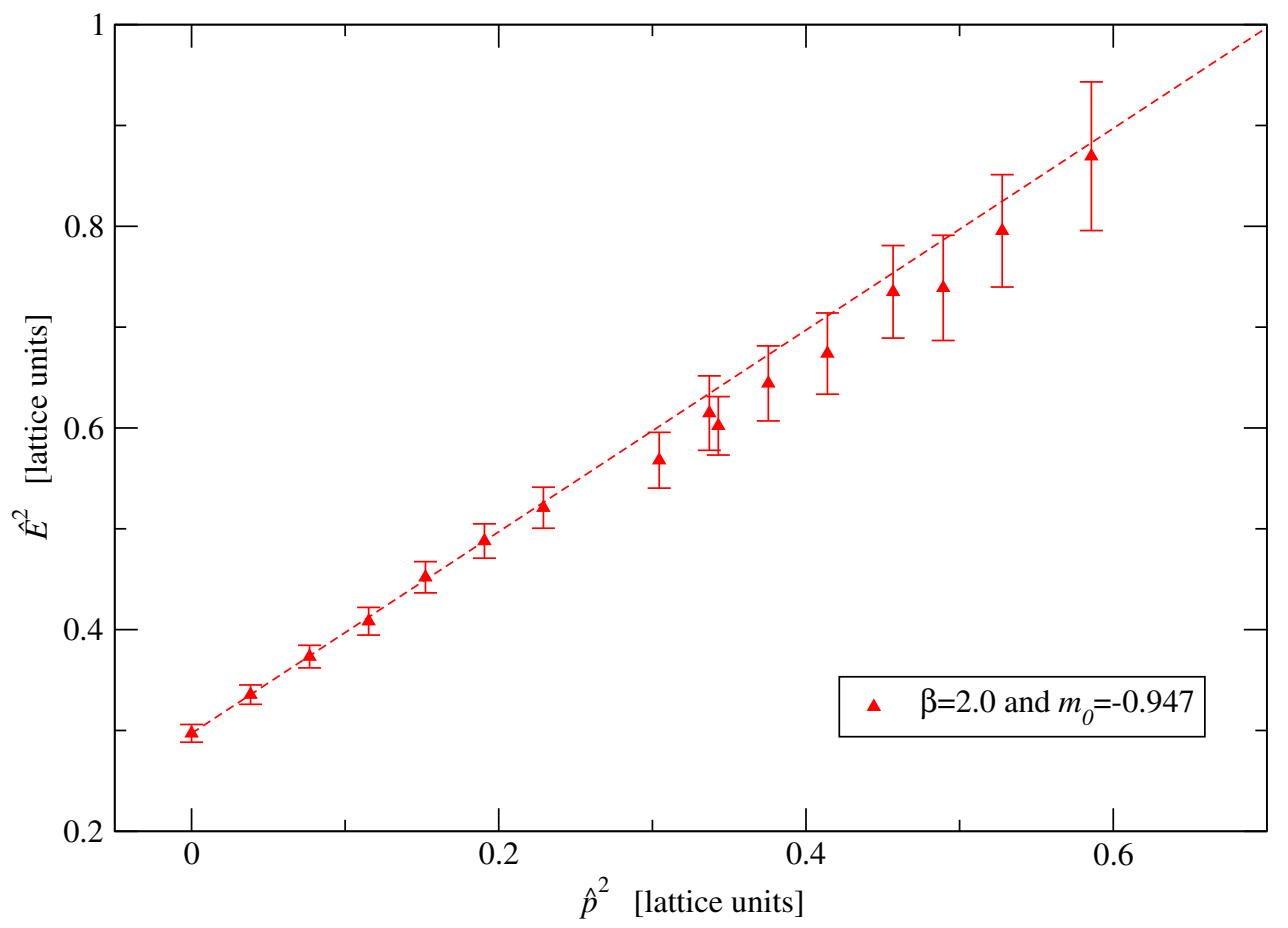

Figure 5. Squared energy of the vector meson as a function of $\hat{p}^{2}=4 \sin ^{2}\left(p_{x} / 2\right)+4 \sin ^{2}\left(p_{y} / 2\right)+$ $4 \sin ^{2}\left(p_{z} / 2\right)$, for $\left(\beta, m_{0}\right)=(2.0,-0.947)$. The straight line is $4 \sinh ^{2}\left(m_{V} / 2\right)+\hat{p}^{2}$ for the measured lattice mass $m_{V}$.

Any difference between the lattice and continuum expressions is due to discretization errors that are small for our ensembles.

Numerical results for the form factor at $\left(\beta, m_{0}\right)=(2.2,-0.72)$ are shown in figure 7 . The lattice data have the shape of a simple vector meson pole, but with a mass parameter significantly different from the lattice vector meson mass. As mentioned previously, our coarser lattice has almost the same vector meson mass so it should give essentially the same form factor, and figure 8 verifies this expectation. It too is thus significantly below its vector meson pole.

It is no surprise that the lightest vector meson does not explain the entire Goldstone form factor. For QCD, chiral perturbation theory contains correction terms suppressed by powers of $m_{\pi}^{2} / \Lambda_{\chi}^{2}[39]$ and similar terms are present in our $\mathrm{SU}(2)$ theory. In fact, the $\mathrm{SU}(2)$ theory has five Goldstone bosons instead of only three. If such chiral terms are responsible for the difference between lattice results and the vector pole, then that difference should be reduced when the fermion mass is reduced. Figure 9 supports this view by showing that the vector meson pole is in statistical agreement with lattice results at our lightest fermion mass.

\section{Photon-dark matter form factor: the basics}

To make predictions for experimental searches, we can follow the general framework for technicolor DM developed in [5]. A more elaborate discussion is presented in following sections. Here we give only some basics. 


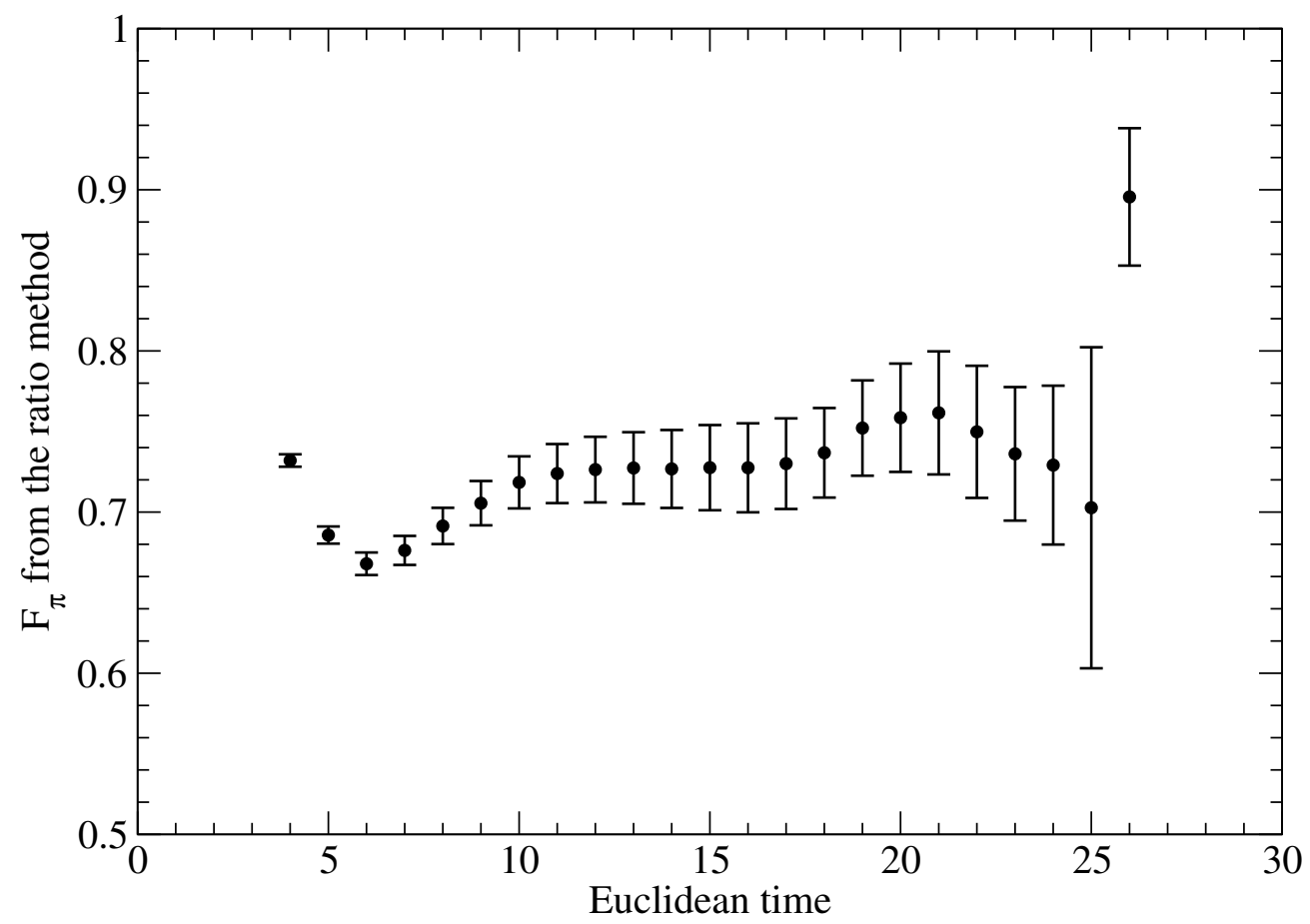

Figure 6. The ratio definition of the Goldstone boson form factor, eq. (2.20), for momentum $\left(k_{x}, k_{y}, k_{z}\right)=(1,1,0)$ in the ensemble having $\left(\beta, m_{0}\right)=(2.2,-0.72)$. The energetic Goldstone is created at $t_{i}=5$, the stationary Goldstone is annihilated at $t_{f}=27$, and the ratio should be fit to a constant for Euclidean times $t$ that satisfy $t_{i} \ll t \ll t_{f}$.

The charge radius of a scalar couples to the photon as follows:

$$
\mathcal{L}_{B}=i e \frac{d_{B}}{\Lambda^{2}} \phi^{*} \overleftrightarrow{\partial_{\mu}} \phi \partial_{\nu} F^{\mu \nu}
$$

In our case we have a specific expression for the coefficient,

$$
\begin{aligned}
\frac{d_{B}}{\Lambda^{2}} & =\lim _{Q^{2} \rightarrow 0} \frac{1}{Q^{2}}\left[\frac{1}{2} \frac{m_{\rho_{u}}^{2}}{m_{\rho_{u}}^{2}+Q^{2}}-\frac{1}{2} \frac{m_{\rho_{d}}^{2}}{m_{\rho_{d}}^{2}+Q^{2}}\right] \\
& =\frac{m_{\rho_{u}}^{2}-m_{\rho_{d}}^{2}}{2 m_{\rho_{u}}^{2} m_{\rho_{d}}^{2}}
\end{aligned}
$$

which, for small isospin breaking $\left(m_{\rho_{u}} \approx m_{\rho_{d}} \equiv m_{\rho}\right)$, corresponds to

$$
\begin{aligned}
\Lambda & =m_{\rho}, \\
d_{B} & =\left(m_{\rho_{u}}-m_{\rho_{d}}\right) / m_{\rho} .
\end{aligned}
$$

For the numerical value of $m_{\rho}$ we use $2.5 \pm 0.5 \mathrm{TeV}$ [15].

Also from [5], the cross section for a DM particle $\phi$ scattering from a nucleon through photon exchange is

$$
\sigma_{p}^{\gamma}=\frac{\mu^{2}}{4 \pi}\left(\frac{8 \pi \alpha d_{B}}{\Lambda^{2}}\right)^{2}
$$




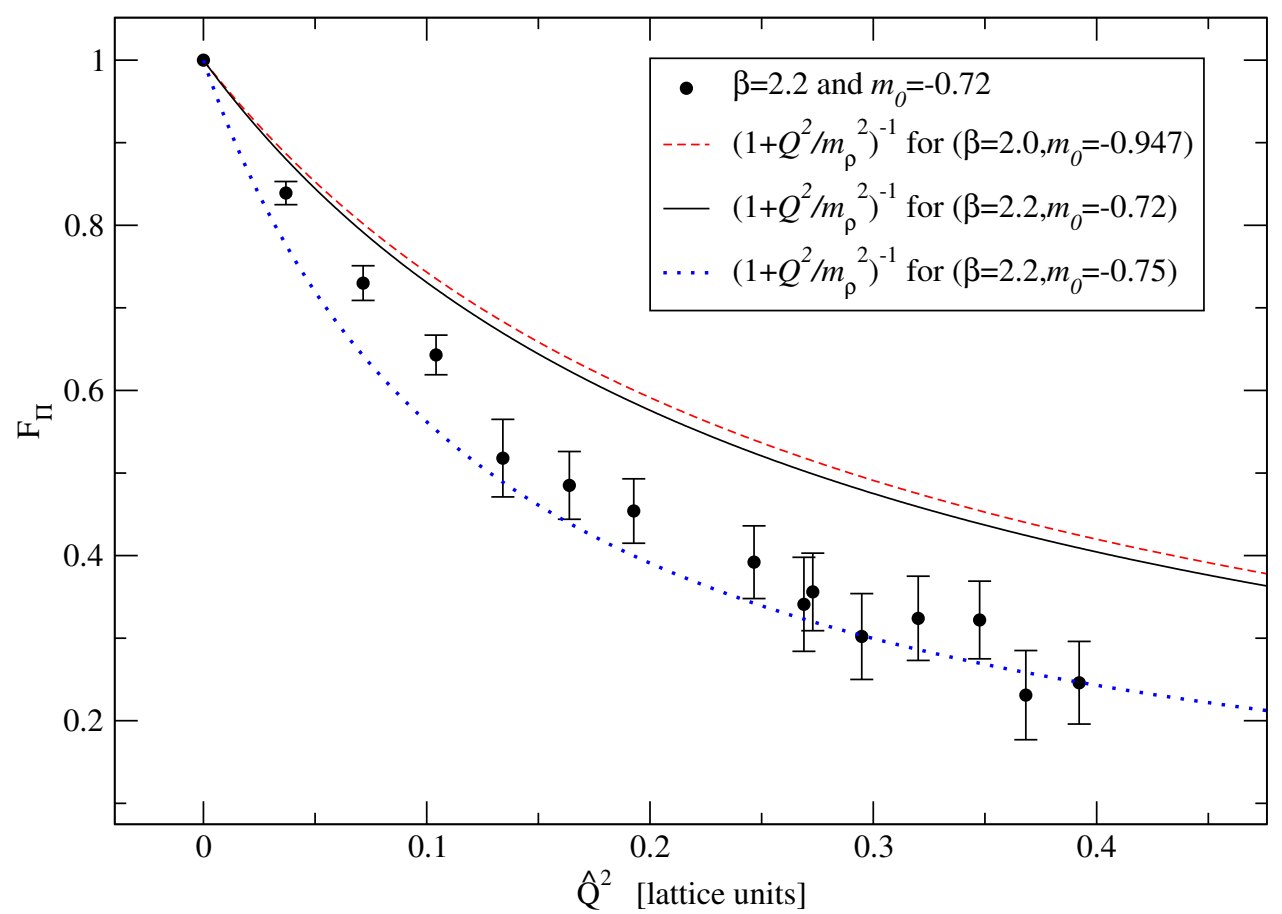

Figure 7. Lattice result for the Goldstone form factor at $\left(\beta, m_{0}\right)=(2.2,-0.72)$. The solid curve is the prediction from a simple vector meson pole with vector mass taken directly from our lattice simulation. The dashed and dotted curves are shown only to aid comparison with figures 8 and 9 .

where $\mu=m_{\phi} m_{N} /\left(m_{\phi}+m_{N}\right)$. Assuming $m_{\phi}>m_{N}$, we see that $m_{N} / 2 \mu<m_{N}$ and the only remaining unknown is $\left|d_{B}\right|$ which is clearly less than unity. We therefore have an upper bound on the cross section in this model, ${ }^{1}$

$$
\sigma_{p}^{\gamma}<2.3 \times 10^{-44} \mathrm{~cm}^{2} .
$$

However, it is important to consider the cross section for scattering through Higgs exchange as well, which can interfere with photon exchange. This issue will be addressed in the upcoming section 6 .

\section{Adding the composite Higgs}

Besides the photon interactions we expect also a composite Higgs exchange $[5,8,11,30]$. The relevant, for detection experiments, Lagrangian terms between our DM candidate and the composite Higgs are

$$
\frac{d_{1}}{\Lambda} h \partial_{\mu} \phi^{*} \partial^{\mu} \phi+\frac{d_{2}}{\Lambda} m_{\phi}^{2} h \phi^{*} \phi .
$$

We have taken into account the pseudo-Goldstone nature of the DM field $\phi$ and therefore we expect $d_{1}$ and $d_{2}$ to be order unity.

\footnotetext{
${ }^{1}$ Note that we could perform the simulations with degenerated fermion masses as the isospin breaking is only parameterized by the small unknown $d_{B}$.
} 


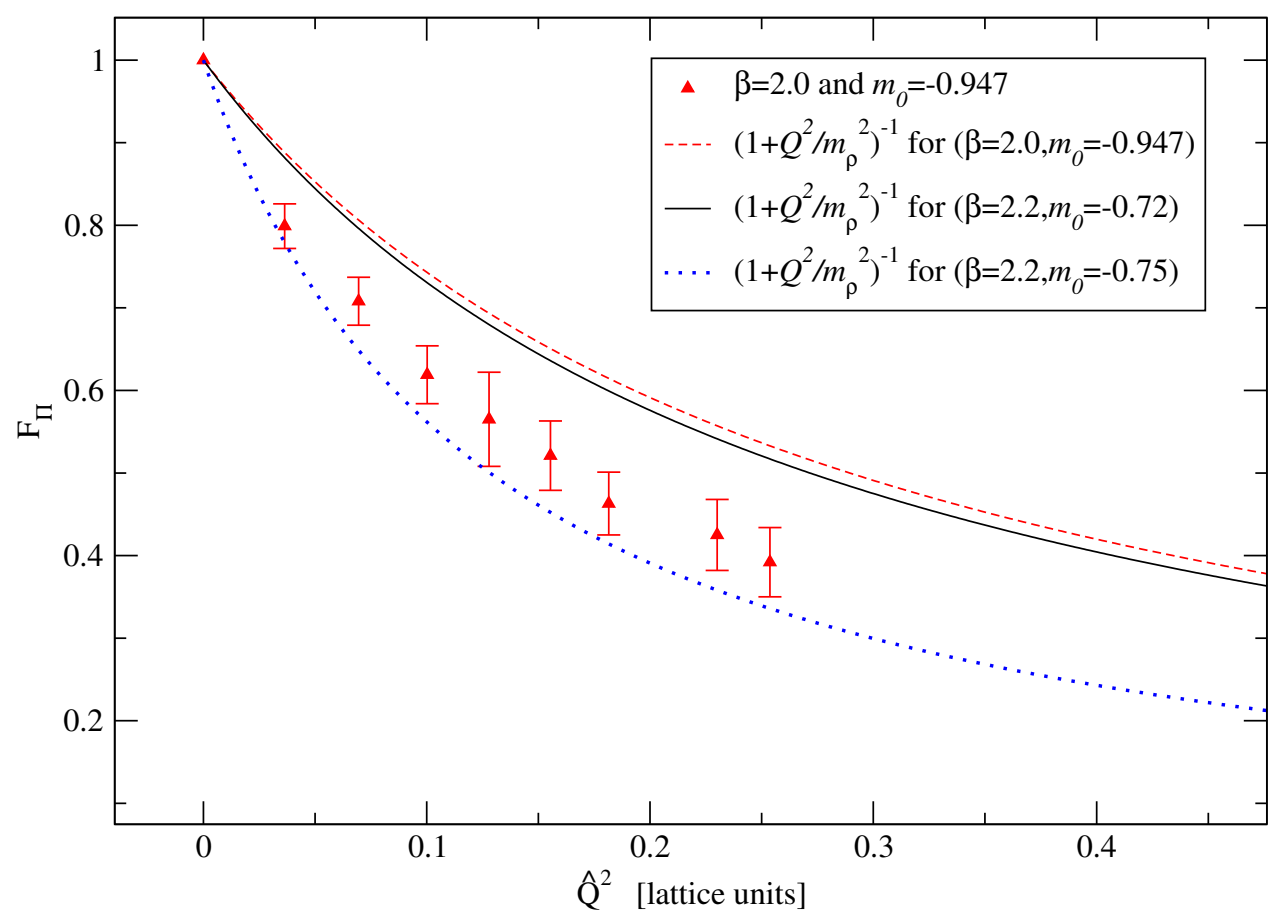

Figure 8. Lattice result for the Goldstone form factor at $\left(\beta, m_{0}\right)=(2.0,-0.947)$. The dashed curve is the prediction from a simple vector meson pole with vector mass taken directly from our lattice simulation. The solid and dotted curves are shown only to aid comparison with figures 7 and 9 .

Making the further minimal assumption that the composite Higgs state couples to the standard model fermions with a strength proportional to their masses, as it is for the ordinary Higgs, the zero momentum transfer cross section of $\phi$ scattering off a nucleus with $Z$ protons and $A-Z$ neutrons is $[5,11]$

$$
\sigma_{A}=\frac{\mu_{A}^{2}}{4 \pi}\left|Z f_{p}+(A-Z) f_{n}\right|^{2}
$$

where

$$
f_{n}=d_{H} f \frac{m_{p}}{m_{H}^{2} m_{\phi}}, \quad f_{p}=f_{n}-\frac{8 \pi \alpha d_{B}}{\Lambda^{2}},
$$

$m_{p}$ is the nucleon mass, $\mu_{A}$ is the $\phi$-nucleus reduced mass and $f \sim 0.3$ parametrizes the Higgs to nucleon coupling and we have defined [30]:

$$
d_{H}=-\frac{d_{1}+d_{2}}{v_{E W} \Lambda} m_{\phi}^{2}
$$

The event rate for generic couplings $f_{n}$ and $f_{p}$ is

$$
R=\sigma_{p} \sum_{i} \eta_{i} \frac{\mu_{A_{i}}^{2}}{\mu_{p}^{2}} I_{A_{i}}\left|Z+\left(A_{i}-Z\right) f_{n} / f_{p}\right|^{2},
$$




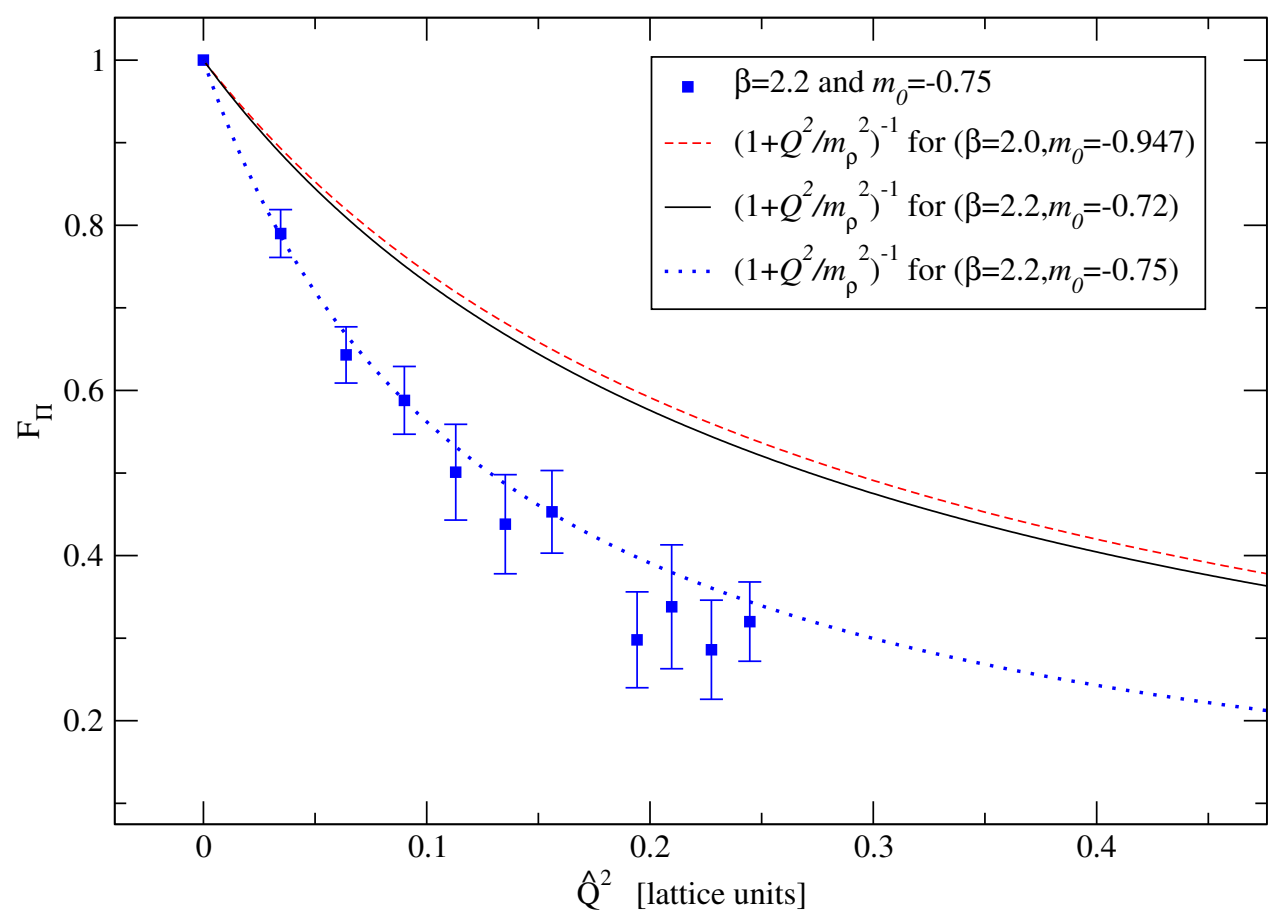

Figure 9. Lattice result for the Goldstone form factor at $\left(\beta, m_{0}\right)=(2.2,-0.75)$. The dotted curve is the prediction from a simple vector meson pole with vector mass taken directly from our lattice simulation. The solid and dashed curves are shown only to aid comparison with figures 7 and 8 .

where $\eta_{i}$ is the abundance of the specific isotope $A_{i}$ in the detector material, and $I_{A_{i}}$ contains all the astrophysical factors as well as the nucleon form factor $F_{A_{i}}\left(E_{R}\right)$. For a given isotope we have

$$
I_{A_{i}}=N_{T} n_{\phi} \int \mathrm{d} E_{R} \int_{v_{\min }}^{v_{\mathrm{esc}}} \mathrm{d}^{3} v f(v) \frac{m_{A_{i}}}{2 v \mu_{A_{i}}^{2}} F_{A_{i}}^{2}\left(E_{R}\right) .
$$

Here $m_{A_{i}}$ is the mass of the target nucleus, $N_{T}$ is the number of target nuclei, $n_{\phi}$ is the local number density of DM particles, and $f(v)$ is their local velocity distribution. The velocity integration is limited between the minimum velocity required in order to transfer a recoil energy $E_{R}$ to the scattered nucleus, $v_{\min }=\sqrt{m_{A} E_{\mathrm{R}} / 2 \mu_{A}^{2}}$, and the escape velocity from the galaxy $v_{\text {esc }}$. The $\phi$-proton cross section $\sigma_{p}=\mu_{p}^{2}\left|f_{p}\right|^{2} / 4 \pi$ can be easily obtained by setting $A=Z=1$ in eq. (6.2).

Direct DM search collaborations quote constraints on generic WIMP-nuclei cross sections normalized to the WIMP-nucleon cross section $\sigma_{p}^{\exp }$ (assuming conventionally $f_{n}=f_{p}$ ). Therefore the experimentally constrained event rate can be cast in the following form

$$
R=\sigma_{p}^{\exp } \sum_{i} \eta_{i} \frac{\mu_{A_{i}}^{2}}{\mu_{p}^{2}} I_{A_{i}} A_{i}^{2} .
$$

Equating eqs. (6.5) and (6.7) yields the experimental constraints on the generic WIMP- 

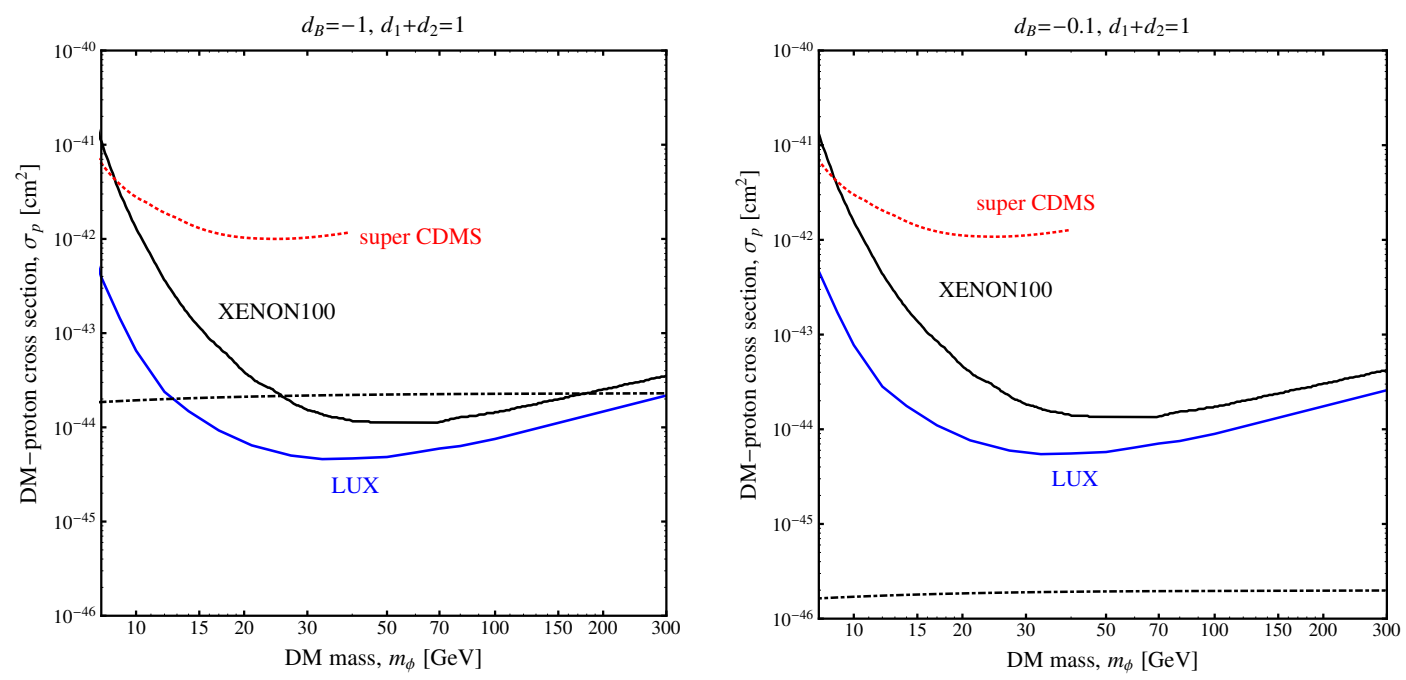

Figure 10. Exclusion contours in the $\left(m_{\phi}, \sigma_{p}\right)$ plane for $d_{B}=-1$ (left panel) and $d_{B}=-0.1$ (right panel). The red-dotted contour is the exclusion plot by Super CDMS [37]; and the black and blue lines are respectively the exclusion plots from Xenon100 [33] and LUX [36] experiments. The composite Goldstone DM cross sections are the black-dot-dashed curves for $d_{1}+d_{2}=1$ in both figures with $d_{B}=-1$ for the left figure and $d_{B}=-0.1$ for the right one.

proton cross section $\sigma_{p}$ with arbitrary couplings $f_{p}$ and $f_{n}$

$$
\sigma_{p}=\sigma_{p}^{\exp } \frac{\sum_{i} \eta_{i} \mu_{A_{i}}^{2} I_{A_{i}} A_{i}^{2}}{\sum_{i} \eta_{i} \mu_{A_{i}}^{2} I_{A_{i}}\left|Z+\left(A_{i}-Z\right) f_{n} / f_{p}\right|^{2}} .
$$

Provided that the factors $I_{A_{i}}$ do not change significantly from one isotope to another as it is the case [30], they drop out from the ratio.

In the left and right panels of figure 10 we plot the exclusion limits from Super CDMS, Xenon100 and LUX in the $\left(m_{\phi}, \sigma_{p}\right)$ plane for $d_{B}=-1$ (left panel) and $d_{B}=-0.1$ (right panel). In both cases we used the value $d_{1}+d_{2}=1$. From the figure we observe that LUX and XENON100 start putting interesting constraints on the composite GB DM parameters for masses between 15 and $300 \mathrm{GeV}$. In particular we cannot have too large values for the isospin breaking parameter $d_{B}$.

\section{Conclusions}

We now summarize the usefulness and limitations of the present work, beginning with the numerical output relevant for experimental dark matter searches, and then discussing lessons applicable to future lattice studies.

Our calculated results for the dark matter-proton cross section are displayed in figure 10. Though both photon exchange and composite Higgs exchange were included, it is important to notice that composite Higgs exchange is essentially negligible in figure 10 . To see this, notice that reducing $\left|d_{B}\right|$ from 1.0 to 0.1 reduces the cross section by about a factor of 100. This is simply the factor of $d_{B}^{2}$ from photon exchange in eq. (5.6). This result holds true for any reasonable choice for the parameters of composite Higgs exchange 
in section 6. Those parameters could become relatively more important as $\left|d_{B}\right|$ shrinks, but will remain far beyond experimental reach for some time.

Our calculation of the cross section depends on vector meson dominance (VMD) providing a reasonable description for the GB form factor. Figure 9 shows that VMD works well for our ensemble that is closest to the continuum limit and chiral limit, while figures 7 and 8 show that the dependences on lattice spacing and on quark mass are consistent with qualitative expectations (see section 4), but of course this is not a rigorous proof of VMD in this theory. Additional lattice studies by other researchers are encouraged. In principle, a direct lattice simulation of the cross section at $m_{u} \neq m_{d}$ could be performed to bypass the need for VMD entirely, but disconnected diagrams would make that very costly. For the present work, we must rely on VMD tested numerically to the extent that our lattice simulations would allow in section 4 .

We find that the theoretical composite GB DM cross section (the black dot-dashed curves of figure 10) is constrained by the most stringent experiments for sufficiently large weak isospin breaking and a composite GB DM mass between 15 and $300 \mathrm{GeV}$. The maximal size of the cross section with ordinary matter, at low energies, is set by having explicitly shown, via lattice simulations, that in this theory the relevant form factors are saturated by a single vector meson exchange whose mass is in the $2.5 \mathrm{TeV}$ energy range.

If the isospin breaking parameter is small one can envision models with larger cross sections. These would require smaller values of the vector masses which can be obtained, for example, by rendering the theory near conformal by either adding new matter gauged under the composite dynamics and singlet with respect to SM interactions [2, 8], and/or changing the matter representation or the composite gauge group [10,61]. Lattice investigations of non-GB composite DM were performed in [26-29].

Numerical results in the present paper are specific to $\mathrm{SU}(2)$ gauge theory with 2 fundamental fermions. More realistic models could be built by extending this in various alternative ways, and of course the cross section will change in each case. Our calculational scheme could be applied to these extended models also, one at a time, by testing VMD and then avoiding disconnected diagrams whenever VMD is satisfied. ${ }^{2}$ We hope this proposed scheme, tested in this paper for the basic template theory, will encourage new lattice studies of interesting candidate theories using the same method.

\section{Acknowledgments}

We thank Ian Shoemaker for discussions and for adding the latest constraints for DM in figure 10. This work was supported in part by the Natural Sciences and Engineering Research Council (NSERC) of Canada, the Danish National Research Foundation DNRF:90 grant, and by a Lundbeck Foundation Fellowship grant. Computing facilities were provided by the Danish Centre for Scientific Computing and Canada's Shared Hierarchical Academic Research Computing Network (SHARCNET: http://www.sharcnet.ca).

\footnotetext{
${ }^{2}$ The complete disconnected study would eventually be warranted for a successful model, but not during the initial explorations.
} 


\section{A Lattice measurements}

In this appendix we list the numbers of the lattice measurements of $F_{\Pi}$ in table 1.

\begin{tabular}{|cccc|}
\hline$\beta$ & $m_{0}$ & $Q^{2}$ & $F_{\Pi}$ \\
\hline 2.0 & -0.947 & 0 & $1(0)$ \\
2.0 & -0.947 & 0.03638 & $0.80(3)$ \\
2.0 & -0.947 & 0.0695 & $0.71(3)$ \\
2.0 & -0.947 & 0.1002 & $0.62(4)$ \\
2.0 & -0.947 & 0.1279 & $0.57(6)$ \\
2.0 & -0.947 & 0.1554 & $0.52(4)$ \\
2.0 & -0.947 & 0.1815 & $0.46(4)$ \\
2.0 & -0.947 & 0.2301 & $0.43(5)$ \\
2.0 & -0.947 & 0.2537 & $0.39(5)$ \\
2.2 & -0.72 & 0 & $1(0)$ \\
2.2 & -0.72 & 0.03698 & $0.839(14)$ \\
2.2 & -0.72 & 0.07154 & $0.73(3)$ \\
2.2 & -0.72 & 0.1042 & $0.64(3)$ \\
2.2 & -0.72 & 0.1341 & $0.52(5)$ \\
2.2 & -0.72 & 0.1639 & $0.49(5)$ \\
2.2 & -0.72 & 0.1927 & $0.45(4)$ \\
2.2 & -0.72 & 0.2466 & $0.39(5)$ \\
2.2 & -0.72 & 0.2729 & $0.37(5)$ \\
\hline
\end{tabular}

\begin{tabular}{|cccc|}
\hline$\beta$ & $m_{0}$ & $Q^{2}$ & $F_{\Pi}$ \\
\hline 2.2 & -0.72 & 0.2689 & $0.34(6)$ \\
2.2 & -0.72 & 0.2948 & $0.30(6)$ \\
2.2 & -0.72 & 0.3201 & $0.32(6)$ \\
2.2 & -0.72 & 0.3476 & $0.32(5)$ \\
2.2 & -0.72 & 0.3683 & $0.23(6)$ \\
2.2 & -0.72 & 0.3922 & $0.24(5)$ \\
2.2 & -0.75 & 0 & $1(0)$ \\
2.2 & -0.75 & 0.0346 & $0.79(3)$ \\
2.2 & -0.75 & 0.0639 & $0.64(4)$ \\
2.2 & -0.75 & 0.09 & $0.58(5)$ \\
2.2 & -0.75 & 0.113 & $0.50(6)$ \\
2.2 & -0.75 & 0.1352 & $0.44(6)$ \\
2.2 & -0.75 & 0.1561 & $0.45(5)$ \\
2.2 & -0.75 & 0.1942 & $0.30(6)$ \\
2.2 & -0.75 & 0.2097 & $0.34(8)$ \\
2.2 & -0.75 & 0.2275 & $0.29(6)$ \\
2.2 & -0.75 & 0.2447 & $0.32(5)$ \\
\hline
\end{tabular}

Table 1. The values for $F_{\Pi}$.

Open Access. This article is distributed under the terms of the Creative Commons Attribution License (CC-BY 4.0), which permits any use, distribution and reproduction in any medium, provided the original author(s) and source are credited.

\section{References}

[1] S. Nussinov, Technocosmology: could a technibaryon excess provide a 'natural' missing mass candidate?, Phys. Lett. B 165 (1985) 55 [InSPIRE].

[2] D.D. Dietrich and F. Sannino, Conformal window of $\mathrm{SU}(N)$ gauge theories with fermions in higher dimensional representations, Phys. Rev. D 75 (2007) 085018 [hep-ph/0611341] [INSPIRE].

[3] E. Nardi, F. Sannino and A. Strumia, Decaying dark matter can explain the $e^{ \pm}$excesses, JCAP 01 (2009) 043 [arXiv:0811.4153] [InSPIRE].

[4] S.B. Gudnason, C. Kouvaris and F. Sannino, Towards working technicolor: effective theories and dark matter, Phys. Rev. D 73 (2006) 115003 [hep-ph/0603014] [INSPIRE]. 
[5] R. Foadi, M.T. Frandsen and F. Sannino, Technicolor dark matter, Phys. Rev. D 80 (2009) 037702 [arXiv:0812.3406] [INSPIRE].

[6] M.Y. Khlopov and C. Kouvaris, Composite dark matter from a model with composite Higgs boson, Phys. Rev. D 78 (2008) 065040 [arXiv:0806.1191] [InSPIRE].

[7] F. Sannino, Conformal dynamics for TeV physics and cosmology, Acta Phys. Polon. B 40 (2009) 3533 [arXiv:0911.0931] [INSPIRE].

[8] T.A. Ryttov and F. Sannino, Ultra minimal technicolor and its dark matter TIMP, Phys. Rev. D 78 (2008) 115010 [arXiv:0809.0713] [INSPIRE].

[9] D.E. Kaplan, M.A. Luty and K.M. Zurek, Asymmetric dark matter, Phys. Rev. D 79 (2009) 115016 [arXiv:0901.4117] [INSPIRE].

[10] M.T. Frandsen and F. Sannino, iTIMP: isotriplet Technicolor Interacting massive particle as dark matter, Phys. Rev. D 81 (2010) 097704 [arXiv:0911.1570] [InSPIRE].

[11] A. Belyaev, M.T. Frandsen, S. Sarkar and F. Sannino, Mixed dark matter from technicolor, Phys. Rev. D 83 (2011) 015007 [arXiv: 1007.4839] [INSPIRE].

[12] D.B. Kaplan and H. Georgi, SU(2) $\times \mathrm{U}(1)$ breaking by vacuum misalignment, Phys. Lett. B 136 (1984) 183 [INSPIRE].

[13] G. Cacciapaglia and F. Sannino, Fundamental composite (Goldstone) Higgs dynamics, JHEP 04 (2014) 111 [arXiv: 1402.0233] [INSPIRE].

[14] R. Lewis, C. Pica and F. Sannino, Light asymmetric dark matter on the lattice: SU(2) technicolor with two fundamental flavors, Phys. Rev. D 85 (2012) 014504 [arXiv:1109.3513] [INSPIRE].

[15] A. Hietanen, R. Lewis, C. Pica and F. Sannino, Fundamental composite Higgs dynamics on the lattice: SU(2) with two flavors, JHEP 07 (2014) 116 [arXiv: 1404.2794] [INSPIRE].

[16] R. Foadi, M.T. Frandsen and F. Sannino, $125 \mathrm{GeV}$ Higgs boson from a not so light technicolor scalar, Phys. Rev. D 87 (2013) 095001 [arXiv:1211.1083] [INSPIRE].

[17] T. Appelquist et al., Lattice gauge theories at the energy frontier, arXiv:1309.1206 [INSPIRE].

[18] T. Appelquistet al., Two-color theory with novel infrared behavior, Phys. Rev. Lett. 112 (2014) 111601 [arXiv:1311.4889] [INSPIRE].

[19] K.-i. Nagai, G. Carrillo-Ruiz, G. Koleva and R. Lewis, Exploration of SU $\left(N_{c}\right)$ gauge theory with many Wilson fermions at strong coupling, Phys. Rev. D 80 (2009) 074508 [arXiv:0908.0166] [INSPIRE].

[20] S. Hands, S. Kim and J.-I. Skullerud, Deconfinement in dense 2-color QCD, Eur. Phys. J. C 48 (2006) 193 [hep-lat/0604004] [INSPIRE].

[21] S. Hands, P. Sitch and J.-I. Skullerud, Hadron spectrum in a two-colour baryon-rich medium, Phys. Lett. B 662 (2008) 405 [arXiv:0710.1966] [INSPIRE].

[22] S. Hands, S. Kim and J.-I. Skullerud, A quarkyonic phase in dense two color matter?, Phys. Rev. D 81 (2010) 091502 [arXiv: 1001.1682] [INSPIRE].

[23] S. Hands and P. Kenny, Topological fluctuations in dense matter with two colors, Phys. Lett. B 701 (2011) 373 [arXiv:1104.0522] [INSPIRE].

[24] S. Cotter, P. Giudice, S. Hands and J.-I. Skullerud, Towards the phase diagram of dense two-color matter, Phys. Rev. D 87 (2013) 034507 [arXiv:1210.4496] [INSPIRE]. 
[25] H. Matsufuru, Y. Kikukawa, K.-i. Nagai and N. Yamada, Lattice simulation of SU(2) gauge theory with chirally symmetric fermions, PoS (LATTICE 2013) 123 [arXiv:1401.6655] [INSPIRE].

[26] W. Detmold, M. McCullough and A. Pochinsky, Dark nuclei I: cosmology and indirect detection, arXiv:1406.2276 [INSPIRE].

[27] W. Detmold, M. McCullough and A. Pochinsky, Dark nuclei II: nuclear spectroscopy in two-colour QCD, arXiv:1406.4116 [INSPIRE].

[28] Lattice Strong Dynamics (LSD) collaboration, T. Appelquist et al., Lattice calculation of composite dark matter form factors, Phys. Rev. D 88 (2013) 014502 [arXiv:1301.1693] [INSPIRE].

[29] Lattice Strong Dynamics (LSD) collaboration, T. Appelquist et al., Composite bosonic baryon dark matter on the lattice: $\mathrm{SU}(4)$ baryon spectrum and the effective Higgs interaction, Phys. Rev. D 89 (2014) 094508 [arXiv: 1402.6656] [inSPIRE].

[30] E. Del Nobile, C. Kouvaris and F. Sannino, Interfering composite asymmetric dark matter for DAMA and CoGeNT, Phys. Rev. D 84 (2011) 027301 [arXiv:1105.5431] [INSPIRE].

[31] E. Del Nobile, C. Kouvaris, F. Sannino and J. Virkajarvi, Dark matter interference, Mod. Phys. Lett. A 27 (2012) 1250108 [arXiv:1111.1902] [INSPIRE].

[32] DAMA collaboration, R. Bernabei et al., First results from DAMA/LIBRA and the combined results with DAMA/NaI, Eur. Phys. J. C 56 (2008) 333 [arXiv:0804.2741] [INSPIRE].

[33] XENON100 collaboration, E. Aprile et al., Dark matter results from 100 live days of XENON100 data, Phys. Rev. Lett. 107 (2011) 131302 [arXiv:1104.2549] [INSPIRE].

[34] XENON100 collaboration, E. Aprile et al., Dark matter results from 225 live days of XENON100 data, Phys. Rev. Lett. 109 (2012) 181301 [arXiv:1207.5988] [INSPIRE].

[35] CDMS-II collaboration, Z. Ahmed et al., Dark matter search results from the CDMS II experiment, Science 327 (2010) 1619 [arXiv:0912.3592] [INSPIRE].

[36] LUX collaboration, D.S. Akerib et al., First results from the LUX dark matter experiment at the Sanford Underground Research Facility, Phys. Rev. Lett. 112 (2014) 091303 [arXiv: 1310.8214] [INSPIRE].

[37] SuPERCDMS collaboration, R. Agnese et al., Search for low-mass weakly interacting massive particles with SuperCDMS, Phys. Rev. Lett. 112 (2014) 241302 [arXiv:1402.7137] [INSPIRE].

[38] Lattice Hadron Physics collaboration, F.D.R. Bonnet, et al., Lattice computations of the pion form-factor, Phys. Rev. D 72 (2005) 054506 [hep-lat/0411028] [INSPIRE].

[39] J. Gasser and H. Leutwyler, Chiral perturbation theory: expansions in the mass of the strange quark, Nucl. Phys. B 250 (1985) 465 [INSPIRE].

[40] QCDSF/UKQCD collaboration, D. Brommel et al., The pion form-factor from lattice QCD with two dynamical flavours, Eur. Phys. J. C 51 (2007) 335 [hep-lat/0608021] [InSPIRE].

[41] ETM collaboration, R. Frezzotti et al., Electromagnetic form factor of the pion from twisted-mass lattice QCD at $N_{f}=2$, Phys. Rev. D 79 (2009) 074506 [arXiv:0812.4042] [INSPIRE].

[42] P.A. Boyle et al., The pion's electromagnetic form-factor at small momentum transfer in full lattice QCD, JHEP 07 (2008) 112 [arXiv:0804.3971] [INSPIRE]. 
[43] JLQCD Collaboration, TWQCD collaboration, S. Aoki et al., Pion form factors from two-flavor lattice QCD with exact chiral symmetry, Phys. Rev. D 80 (2009) 034508 [arXiv: 0905.2465] [INSPIRE].

[44] O.H. Nguyen, K.-I. Ishikawa, A. Ukawa and N. Ukita, Electromagnetic form factor of pion from $N_{f}=2+1$ dynamical flavor QCD, JHEP 04 (2011) 122 [arXiv:1102.3652] [INSPIRE].

[45] B.B. Brandt, A. Juttner and H. Wittig, Calculation of the pion electromagnetic form factor from lattice $Q C D$, arXiv:1109.0196 [INSPIRE].

[46] B.B. Brandt, A. Jüttner and H. Wittig, The pion vector form factor from lattice $Q C D$ and NNLO chiral perturbation theory, JHEP 11 (2013) 034 [arXiv:1306.2916] [INSPIRE].

[47] W. Wilcox and R.M. Woloshyn, Lattice results on the meson electric form-factor, Phys. Rev. Lett. 54 (1985) 2653 [INSPIRE].

[48] R.M. Woloshyn and A.M. Kobos, Numerical study of the lattice meson form-factor, Phys. Rev. D 33 (1986) 222 [INSPIRE].

[49] R.M. Woloshyn, Lattice meson electric form-factor using Wilson fermions, Phys. Rev. D 34 (1986) 605 [INSPIRE].

[50] G. 't Hooft, A planar diagram theory for strong interactions, Nucl. Phys. B 72 (1974) 461 [INSPIRE].

[51] E. Witten, Baryons in the 1/n expansion, Nucl. Phys. B 160 (1979) 57 [InSPIRE].

[52] P. Masjuan, E. Ruiz Arriola and W. Broniowski, Meson dominance of hadron form factors and large-Nc phenomenology, Phys. Rev. D 87 (2013) 014005 [arXiv:1210.0760] [InSPIRE].

[53] Particle Data Group collaboration, J. Beringer et al., Review of particle physics, Phys. Rev. D 86 (2012) 010001 [INSPIRE].

[54] L. Del Debbio, A. Patella and C. Pica, Higher representations on the lattice: Numerical simulations. SU(2) with adjoint fermions, Phys. Rev. D 81 (2010) 094503 [arXiv:0805.2058] [INSPIRE].

[55] S. Chang, J. Liu, A. Pierce, N. Weiner and I. Yavin, CoGeNT Interpretations, JCAP 08 (2010) 018 [arXiv: 1004.0697] [InSPIRE].

[56] J.L. Feng, J. Kumar, D. Marfatia and D. Sanford, Isospin-violating dark matter, Phys. Lett. B 703 (2011) 124 [arXiv:1102.4331] [INSPIRE].

[57] C. Savage, G. Gelmini, P. Gondolo and K. Freese, XENON10/100 dark matter constraints in comparison with CoGeNT and DAMA: examining the Leff dependence, Phys. Rev. D 83 (2011) 055002 [arXiv: 1006.0972] [INSPIRE].

[58] N. Bozorgnia, G.B. Gelmini and P. Gondolo, Channeling in direct dark matter detection I: channeling fraction in NaI (Tl) crystals, JCAP 11 (2010) 019 [arXiv:1006.3110] [INSPIRE].

[59] CDMS-II collaboration, Z. Ahmed et al., Results from a low-energy analysis of the CDMS II germanium data, Phys. Rev. Lett. 106 (2011) 131302 [arXiv:1011.2482] [INSPIRE].

[60] XENON10 collaboration, J. Angle et al., A search for light dark matter in XENON10 data, Phys. Rev. Lett. 107 (2011) 051301 [Erratum ibid. 110 (2013) 249901] [arXiv:1104.3088] [INSPIRE].

[61] A. Hietanen, C. Pica, F. Sannino and U.I. Sondergaard, Orthogonal technicolor with isotriplet dark matter on the lattice, Phys. Rev. D 87 (2013) 034508 [arXiv:1211.5021] [InSPIRE]. 